\title{
Symmetrization of various friction models based on an Augmented Lagrangian approach
}

Alexander Konyukhov, Karl Schweizerhof Universität Karlsruhe, Institut für Mechanik

Institut für Mechanik

Kaiserstr. 12, Geb. 20.30

76128 Karlsruhe

Tel.: +49 (0) 721/ 608-2071

Fax: +49 (0) 721/ 608-7990

E-Mail: ifm@uni-karlsruhe.de

www.ifm.uni-karlsruhe.de 


\title{
Symmetrization of various friction models based on an Augmented Lagrangian approach.
}

\author{
Alexander Konyukhov, Karl Schweizerhof
}

2006

\section{Abstract}

The standard implementation of the classical Coulomb friction model together with the Newton iterative method for the finite element method leads to nonsymmetric tangent matrices for sliding zones of contact surfaces. This fact is known in literature as consequence of the non-associativity of the friction law. Considering anisotropic models for friction, especially including coupling of adhesion and friction, leads to additional non-symmetries due to anisotropy. Since, non-symmetry of matrices is a non-desirable feature of most engineering problems, various proposals for symmetrization are known in computational mechanics. A further suggestion is made in this contribution. The covariant approach for both isotropic and anisotropic frictional contact problems leads to a very simple structure of the tangent matrices. This allows to obtain very robust tangent matrices within the symmetrized Augmented Lagrangian method. In the current contribution, the nested Uzawa algorithm is applied for symmetrization within the Augmented Lagrangian approach for an anisotropic friction model including adhesion and friction. The numerical examples show the good convergence behavior for various problems such as small and large sliding problems.

\section{Introduction}

The penalty method for frictional contact problems [1], [2] currently is among the most popular schemes in finite element packages, leads to the satisfaction of the contact constraints, such as non-penetration and sticking conditions, only within a certain tolerance. This tolerance is defined by the penalty parameters for both normal and tangential direction. As is known, the classical method of Lagrangian multipliers leads to an exact satisfaction of contact constraints, however, one should take care of the number of multipliers due to the often overstiff behavior of contact interfaces, see e.g. in [3]. Additional degrees of freedoms for the contact tractions are often mentioned among the disadvantages of this method. Recently, various combinations of the Mortar method 
have been developed in e.g. [4], [5], [6] allowing to overcome overstiff behavior especially in the case where contact tractions are computed point-wise at integration points. In addition, good results for the patch-test have been shown also in [3] exploiting the, so-called, segment-to-segment approach, coinciding with the Mortar method with penalty descriptions of the contact traction. Socalled dual Lagrange multipliers have been developed for non-frictional contact in Wohlmuth [7] allowing to condense degrees of freedom for contact traction. Using this approach, the frictional constraints should be carefully treated as a sequence of the Tresca type friction model, see [8]. This approach is similar to the Augmented Lagrangian approach allowing to satisfy the contact constraints for non-penetration and sticking within the nested algorithm. The method is described theoretically in Bertsekas [9] and Fortin and Glowinski [10]. Pietrzak and Curnier [11] developed the Augmented Lagrangian approach for frictional contact including the corresponding saddle point functional. Laursen and Simo [12] proposed a symmetrization procedure based on the nested Uzawa algorithm. This approach is based on symmetrization of the corresponding tangent matrices for the solution of the equilibrium equations, while the contact constraints have been still iteratively satisfied within the external loop with a specified tolerance.

In the current contribution, we will describe the symmetric Augmented Lagrangian method for the coupled anisotropic contact model including anisotropy for friction and adhesion as developed in Konyukhov and Schweizerhof [13], [14]. The geometrical structure of the corresponding tangent matrices allows us to construct very simple symmetric tangent matrices for the anisotropic case. The isotropic case can then be defined as a reduction of the anisotropic case. Good convergence rates are illustrated in the numerical examples.

\section{Covariant description of the coupled anisotropic friction model}

We shortly present here the main details of the coupled interface contact model including anisotropy for adhesion and friction and refer to [13], [14] for further details and corresponding derivations.

At the beginning, a local surface coordinate system is introduced as

$$
\mathbf{r}_{\mathrm{S}}\left(\xi^{1}, \xi^{2}, \xi^{3}\right)=\boldsymbol{\rho}\left(\xi^{1}, \xi^{2}\right)+\mathbf{n} \xi^{3},
$$

where $\xi^{1}, \xi^{2}$ are two convective coordinates and responsible for the tangential contact interaction. The third coordinate $\xi^{3}$ is the value of the penetration and is used to define the properties of the normal interaction

$$
\xi^{3}=\left(\mathbf{r}_{\mathrm{s}}-\boldsymbol{\rho}\right) \cdot \mathbf{n} .
$$


The vector of contact tractions $\mathbf{R}$ is defined as a covariant vector and, therefore, is expressed via the contravariant basis vectors $\boldsymbol{\rho}^{i}$ and $\mathbf{n}$ in the coordinate system (1) as sum of the tangential and normal components

$$
\mathbf{R}=\mathbf{T}+\mathbf{N}=T_{i} \boldsymbol{\rho}^{i}+N \mathbf{n} .
$$

For the generalization into anisotropy for adhesion, the tangential traction vector $T_{i} \boldsymbol{\rho}^{i}$ and the normal traction vector $N \mathbf{n}$ are assumed to be decoupled. Therefore, the generalized constitutive equations for tangential tractions are taken in rate form as

$$
\frac{d \mathbf{T}}{d t}=\mathbf{B}\left(\mathbf{v}_{\mathrm{s}}-\mathbf{v}\right)
$$

where $\mathbf{B}$ is the anisotropic adhesion tensor. The covariant differentiation operations are involved throughout whenever the rate form is given.

The constitutive equation for normal traction is given in closed form and can be viewed as a simple penalty regularization procedure for this normal traction $N$

$$
N=\epsilon_{N} \xi^{3},
$$

where $\epsilon_{N}$ is a parameter of normal compliance, or a penalty parameter.

The anisotropy for friction is chosen as a model of Coulomb type involving proportionality of the frictional force to the normal traction $N$. The corresponding yield function is written as

$$
\Phi=\sqrt{f^{i j} T_{i} T_{j}}-|N|=\sqrt{\mathbf{T} \cdot \mathbf{F T}}-|N|,
$$

where $\mathbf{F}=f^{i j} \boldsymbol{\rho}_{i} \boldsymbol{\rho}_{j}$ is the anisotropic friction tensor.

\subsection{Incremental formulation of the coupled anisotropic model}

Though, initially the model is formulated in the continuous rate form, it has been transformed into incremental form for the final computational model via the application of the backward Euler scheme.

i) The full displacement vector $\Delta \boldsymbol{\xi}=\boldsymbol{\xi}^{(n+1)}-\boldsymbol{\xi}^{(n)}$ is decomposed additively into an elastic increment $\Delta \boldsymbol{\xi}^{e l}$ and into a sliding increment $\Delta \boldsymbol{\xi}^{s l}$ :

$$
\Delta \boldsymbol{\xi}=\Delta \boldsymbol{\xi}^{e l}+\Delta \boldsymbol{\xi}^{s l},
$$

where both vectors are defined in the surface metrics, namely,

$$
\Delta \boldsymbol{\xi}:=\Delta \xi^{i} \boldsymbol{\rho}_{i}=\left(\xi_{(n+1)}^{i}-\xi_{(n)}^{i}\right) \boldsymbol{\rho}_{i} .
$$


ii) The trial elastic, or adhesion force $\mathbf{T}_{(n+1)}^{t r}$ is computed via the incremental evolution equations:

$$
\mathbf{T}_{(n+1)}^{t r}-\mathbf{T}_{(n)}=\mathbf{B}^{(n+1)}\left(\boldsymbol{\xi}_{(n+1)}^{e l}-\boldsymbol{\xi}_{(n)}^{e l}\right) .
$$

iii) The final result if the tangential traction $\mathbf{T}$ is the elastic one (belongs to the adhesion region), or the plastic one if provided by the yield condition of the Coulomb type in each load step becomes:

$$
\begin{aligned}
\Phi^{t r}: & =\sqrt{\mathbf{T}_{(n+1)}^{t r} \cdot \mathbf{F}^{(n+1)} \mathbf{T}_{(n+1)}^{t r}}-\left|N_{(n+1)}\right| \\
& =\sqrt{f^{i j} T_{i(n+1)}^{t r} T_{j(n+1)}^{t r}}-\left|N_{(n+1)}\right|,
\end{aligned}
$$

- If $\Phi^{t r}<0$ then the trial force is a real sticking force $\mathbf{T}=\mathbf{T}^{t r}$.

- If $\Phi^{t r} \geq 0$ then the sliding force must be obtained via the maximum of the energy dissipation function given in the incremental form.

iv) All contact parameters such as sliding traction and sliding distance should be derived via the principle of the maximum dissipation

$$
D^{(n+1)}:=\Delta \boldsymbol{\xi}^{s l} \cdot \mathbf{T}_{(n+1)}^{s l}=\Delta \xi_{s l}^{i} T_{i(n+1)}^{s l}, \quad D^{(n+1)} \longrightarrow \max .
$$

Using the necessary optimization conditions for the functional $D^{(n+1)}$ together with the Kuhn-Tucker conditions from iii) the closed form for the sliding force $\mathbf{T}^{s l}$ is obtained as:

$$
\mathbf{T}^{s l}=-\frac{\mathbf{B F T}^{t r}}{\sqrt{\mathrm{BFT}^{t r} \cdot \mathbf{F B F T}^{t r}}}|N|
$$

\section{Linearization process and structure of matrices}

Since the frictional problem is nonlinear, an iterative solution based on a Newton scheme should be applied. The important part of the implementation is then a consistent tangent matrix which differs for sticking and sliding according to the return-mapping scheme. We present here the results with particular focus on the structure of the matrices especially their symmetry. According to numerical experiences reported in [15], [16]. we will also exclude the curvature parts of the matrices. 


\subsection{Linearization of the normal part $\delta W_{c}^{N}$}

We denote $D(f)$ as a linearization operator acting on a functional $f$ in the covariant form. Thus, linearization of the virtual work of the contact normal traction $N$ is given as:

$$
\begin{gathered}
D\left(\delta W_{c}^{N}\right)=D\left(\int_{s} N \delta \xi^{3} d s\right)=-\underbrace{\int_{s} \epsilon_{N}\left(\delta \mathbf{r}_{s}-\delta \boldsymbol{\rho}\right) \cdot(\mathbf{n} \otimes \mathbf{n})\left(\mathbf{v}_{s}-\mathbf{v}\right) d s}_{\text {leads to } \mathbf{K}_{N}^{m}} \\
-\underbrace{\int_{s} \epsilon_{N} \xi^{3}\left(\delta \boldsymbol{\rho}_{, j} \cdot a^{i j}\left(\mathbf{n} \otimes \boldsymbol{\rho}_{i}\right)\left(\mathbf{v}_{s}-\mathbf{v}\right)+\left(\delta \mathbf{r}_{s}-\delta \boldsymbol{\rho}\right) \cdot a^{i j}\left(\boldsymbol{\rho}_{j} \otimes \mathbf{n}\right) \mathbf{v}_{, i}\right) d s}_{\text {leads to } \mathbf{K}_{N}^{r}},
\end{gathered}
$$

where $a^{i j}$ are contravariant components of the metric tensor for the master surface and $\boldsymbol{\rho}_{j}=\frac{\partial \boldsymbol{\rho}}{\partial \xi^{j}}, j=1,2$ are covariant basis (tangent) vectors. Here, the first term (resp. the second term) after approximation of the geometry leads to the main part of the contact matrix $\mathbf{K}_{N}^{m}$ (resp. the rotational part of the contact matrix $\mathbf{K}_{N}^{r}$ ).

All parts can be algorithmically implemented for any order of approximation. In order to do this only the operator $\mathbf{A}$ for the approximation of the surface geometry has to be introduced. The derivatives with respect to convective coordinates $\mathbf{A}_{\xi}$ are also necessary. Thus, the approximation can be written as

$$
\mathbf{r}_{s}-\boldsymbol{\rho}=\mathbf{A}\{\mathbf{x}\}, \quad \boldsymbol{\rho}_{\xi}=\mathbf{A}_{\xi}\{\mathbf{x}\}
$$

where $\mathbf{x}$ is a nodal vector for the standard FE implementation, or a control points (knots) vector for a CAD approximation. This leads e.g. to the following structure of the contact matrix $\mathbf{K}_{N}^{m}$

$$
\mathbf{K}_{N}^{m}=-\int_{s} \epsilon_{N} \mathbf{A}^{T} \cdot(\mathbf{n} \otimes \mathbf{n}) \mathbf{A} d s,
$$

where the integral is computed via the set of Gauss points defined on the slave segment and penetrating into the master surface (so-called penalty based Mortar method, see Fischer and Wriggers [6]).

\subsection{Linearization of the tangential part $\delta W_{c}^{T}$, sticking case}

The sticking case is understood as a case where the tangential traction remains in the elastic region and, therefore, is computed via the evolution equations ii). Linearization leads to 


$$
\begin{gathered}
D\left(\delta W_{c}^{T}\right)=D\left(\int_{s} T_{i}^{t r} \delta \xi^{i} d s\right)= \\
=\underbrace{\int_{s}\left(\delta \mathbf{r}_{s}-\delta \boldsymbol{\rho}\right) \cdot \mathbf{B}\left(\mathbf{v}_{s}-\mathbf{v}\right) d s-}_{\text {leads to } \mathbf{K}_{T, s t}^{m}} \\
\underbrace{-\int_{s} T_{i}\left(\left(\delta \mathbf{r}_{s}-\delta \boldsymbol{\rho}\right) \cdot a^{i l} a^{j k} \boldsymbol{\rho}_{k} \otimes \boldsymbol{\rho}_{l} \mathbf{v}_{j}+\delta \boldsymbol{\rho}_{, j} \cdot a^{i k} a^{j l} \boldsymbol{\rho}_{k} \otimes \boldsymbol{\rho}_{l}\left(\mathbf{v}_{s}-\mathbf{v}\right)\right) d s,}_{\text {leads to } \mathbf{K}_{T, s t}^{r}}
\end{gathered}
$$

\subsection{Linearization of the tangential part $\delta W_{c}^{T}$, sliding case}

The sliding case is understood as a case where the yield condition iii) is fulfilled and, therefore, the tangential traction is computed as a sliding traction given in eqn. (12). The corresponding linearization leads to

$$
\begin{aligned}
& D\left(\delta W_{c}^{T}\right)=D\left(\int_{s} T_{i}^{s l} \delta \xi^{i} d s\right)= \\
& =\underbrace{\int_{s}\left(\left(\delta \mathbf{r}_{s}-\delta \boldsymbol{\rho}\right) \cdot \frac{\epsilon_{N} \mathbf{B F} \mathbf{T}^{t r} \otimes \mathbf{n}}{\sqrt{\mathbf{B F T}^{t r} \cdot \mathbf{F B F T}}}\left(\mathbf{v}_{s}-\mathbf{v}\right)\right) d s}_{\text {leads to } \mathbf{K}_{T, s l}^{m, 1}} \\
& \underbrace{-\int_{s}\left(\left(\delta \mathbf{r}_{s}-\delta \boldsymbol{\rho}\right) \cdot \frac{|N| \mathbf{B F B}}{\sqrt{\mathbf{B F T}^{t r} \cdot \mathbf{F B F T}}}\left(\mathbf{v}_{s}-\mathbf{v}\right)\right) d s}_{\text {leads to } \mathbf{K}_{T, s l}^{m, 2}} \\
& +\underbrace{\int_{s}\left(\left(\delta \mathbf{r}_{s}-\delta \boldsymbol{\rho}\right) \cdot \frac{|N| \mathbf{B F T}^{t r} \otimes(\mathbf{B F B})^{T} \mathbf{F B F T} \mathbf{T}^{t r}}{\left.\sqrt{\left.\left(\mathbf{B F T}^{t r} \cdot \mathbf{F B F T}\right)^{t r}\right)^{3}}\left(\mathbf{v}_{s}-\mathbf{v}\right)\right) d s}\right.}_{\text {leads to } \mathbf{K}_{T, s l}^{m, 3}} \\
& \underbrace{-\int_{s} T_{i}^{s l}\left[\left(\delta \mathbf{r}_{s}-\delta \boldsymbol{\rho}\right) \cdot a^{i l} a^{j k} \boldsymbol{\rho}_{k} \otimes \boldsymbol{\rho}_{l} \mathbf{v}_{j}+\delta \boldsymbol{\rho}_{, j} \cdot a^{i k} a^{j l} \boldsymbol{\rho}_{k} \otimes \boldsymbol{\rho}_{l}\left(\mathbf{v}_{s}-\mathbf{v}\right)\right] d s .}_{\text {leads to } \mathbf{K}_{T, s l}^{r}}
\end{aligned}
$$

Now we can summarize the results concerning the symmetry of the necessary tangent matrices. As expected, all parts concerning non-frictional and sticking contact, namely $\mathbf{K}_{N}^{m}, \mathbf{K}_{N}^{r}, \mathbf{K}_{T, s t}^{m}$ and $\mathbf{K}_{T, s t}^{r}$ are symmetric. Attention should be paid to the sliding tangent matrix, because it contains both, symmetric and nonsymmetric parts: 
$\mathbf{K}_{T, s l}^{m, 1}$ is the first main part due to the coupling of the normal and the sliding tractions. It appears due to linearization of the normal traction $N$ and it is nonsymmetric.

$\mathbf{K}_{T, s l}^{m, 2}$ is the second main part. It appears due to linearization of the normal trial tangential traction $\mathbf{T}^{t r}$ and preserves symmetry.

$\mathbf{K}_{T, s l}^{m, 3}$ is the third main part and nonsymmetric. It appears due to linearization of the complex term $\frac{1}{\sqrt{\mathbf{B F T}^{t r} \cdot \text { FBFT }^{t r}}}$ reflecting the coupling of anisotropy for adhesion and friction.

Finally, $\mathbf{K}_{T, s l}^{r}$ is the rotational, symmetric part reflecting rotation of the master segment, for more details see [17].

\section{Augmented Lagrangian method and symmetric Uzawa algorithm}

The main advantage of the Augmented Lagrangian in comparison to the penalty method is the possibility to select a value of the penalty parameter leading to well conditioned tangent matrices together with the enforcement of the constraint conditions (non penetration and sticking) within a specified tolerance. The method is constructed as a nested algorithm known as Uzawa algorithm and possesses linear convergence. The algorithm using the full consistent tangent matrix is known as exact Uzawa algorithm, while the inexact algorithm is exploiting a somehow simplified matrix, see results on convergence in Stadler [18]. Laursen and Simo [12] developed a symmetrized Uzawa algorithm, where symmetric matrices for the sliding case have been obtained under the assumption that the normal traction remains constant for the solution of the equilibrium equations within an internal loop. This preserves the quadratic rate of convergence for the internal loop. The correct value is enforced then within the external loop where a linear rate of convergence is preserved.

\subsection{Limitations of the Augmented Lagrangian approach for the coupled anisotropic model}

The complexity for a generalization of the discussed algorithm into anisotropic friction especially including anisotropic adhesion is that the sliding tangent matrix is fully nonsymmetric, moreover, the assumption of isotropy together with the constant normal traction would lead only to the rotational part of the tangent matrix. Since, this part has no influence for small displacement problems as well as in the case when the master segment has no rotation, we obtain only zero matrices. This makes it impossible to obtain any solution. Fortunately, 
the covariant approach allows to estimate the influence of the matrices part by part. Thus, we can construct a symmetric algorithm and analyze it numerically.

Another problem for the coupled anisotropy is that the sticking case is defined when the slave contact point lays inside the elliptic adhesion domain, see Fig. 1. However, as is known from numerical results the ratio $\frac{a}{b}=\frac{\mu_{1} \varepsilon_{2}}{\mu_{2} \varepsilon_{1}}$ does not influence the convergence result in the penalty based approach and can lead to correct kinematics even for large sliding problems. An enforcement to put a slave contact point inside the adhesion ellipse via the Augmented Lagrangian approach necessarily leads to dis-convergence in cases as $\frac{a}{b} \rightarrow 0$, or $\frac{a}{b} \rightarrow \infty$. In computation due to the linear convergence of the Augmented Lagrangian method the global number of iteration is proportional to the ratio $\frac{a}{b}$ for $a>b$, e.g. a simply computable case with $\frac{a}{b}=10$ would lead to a 10-times increase of the global number of iterations for the Augmented Lagrangian method. Thus, one should judge the coupled anisotropic model as an interface model for tangential traction, rather than a penalty based approach.

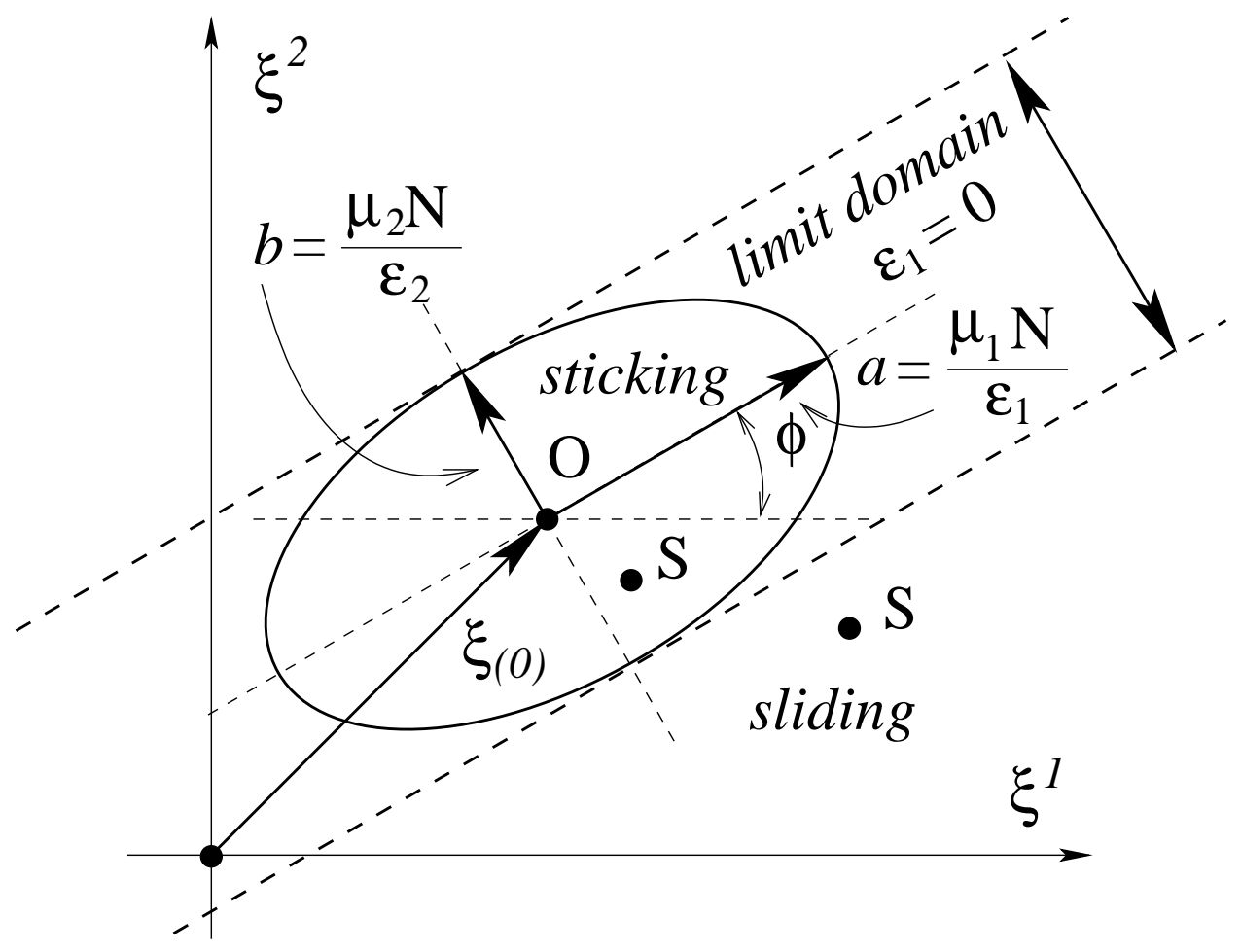

Figure 1: Allowable elastic region (adhesion domain).

Summarizing the discussion, we can define computable cases for the Augmented Lagrangian method in application to the coupled anisotropic model for:

1. Small sliding problems with low anisotropy. Small sliding can be numerically defined as a case where it is important to compute the distribution of the 


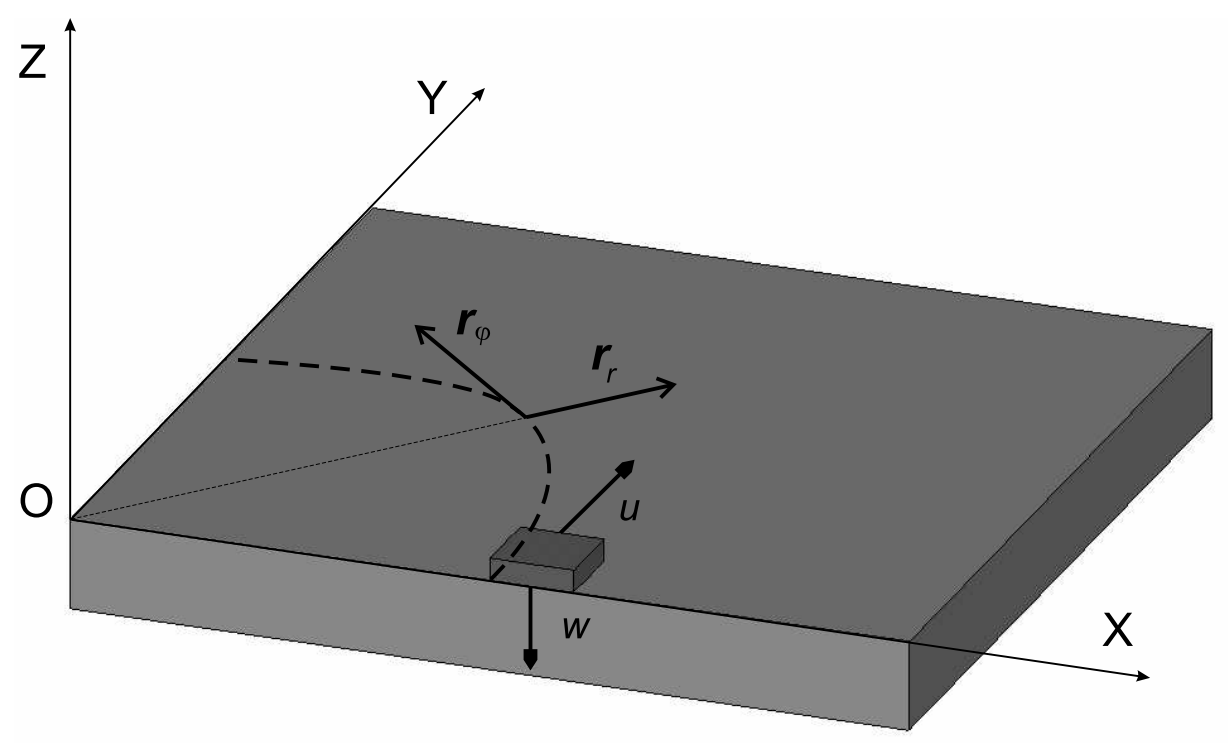

Figure 2: Geometry and loading for the case of polar orthotropy.

sticking-sliding zone. The low anisotropy is defined then as a case with $\frac{a}{b} \approx 1$.

In this case, Lagrangian multipliers for both normal $N$, and tangential traction $\mathbf{T}$ are augmented. The convergence for tangential displacements is checked by the proximity to the initial contact point:

$$
\sqrt{a_{i j}\left(\xi_{(I)}^{i}-\xi_{(K-1)}^{i}\right)\left(\xi_{(I)}^{j}-\xi_{(K-1)}^{j}\right)} \leq \epsilon_{T},
$$

where $\epsilon_{T}$ is a specified tolerance for tangential displacements. The convergence for normal displacements is checked as in the standard isotropic case by the normal penetration

$$
\left|\xi^{3}\right| \leq \epsilon_{N},
$$

where $\epsilon_{N}$ is a specified tolerance for the normal penetration.

2. Large sliding problem with arbitrary anisotropy. Large sliding can be numerically defined as a case where sliding is reached within a single load step. In this case, the global kinematical behavior of a contacting body is of interest rather than the distribution of the sticking-sliding zone.

In this case, the Lagrangian multiplier only for the normal traction $N$ needs to be updated because the tolerance of the sticking condition will normally not influence the tolerance of the computed trajectory. Thus, convergence is checked only for the normal displacement. 
Table 1: Update scheme for normal traction $N$

1. Loop over applied in load incremental steps: $K=K+1$

initialization of Lagrange multiplier $\lambda_{N}^{0}=0$

2. Loop over augmented multipliers: $L=L+1$
\begin{tabular}{|} 
3. Iterative solution of \\
global equilibrium equations: \\
with normal force \\
$N^{K, L, I}=\lambda_{N}^{L-1}+\varepsilon_{N} \xi_{I}^{3}$
\end{tabular}
penetration $\xi_{I}^{3}$ is computed in each iteration $I$.

Update multipliers

$$
\lambda_{N}^{L}=N^{K, L, I}
$$

Convergence is checked by the non-penetration condition:

$$
\left|\xi_{I}^{3}\right| \leq \epsilon_{N}, \text { for } N^{K, L, I}<0,
$$

where $\epsilon_{N}$ - specified tolerance for normal displacements

The symmetrized algorithm in accordance with the inexact Uzawa approach which appears to be numerically effective for the current anisotropic problem is constructed as follows. The internal loop, No. 3 in Table 1 and 2, serves for the solution of the equilibrium equations. The normal traction $N^{K, L, I}$ is computed via the augmented scheme and updated inside loop 2 . The trial tangential traction $\mathbf{T}^{K, L, I}$ for small sliding problems is computed via the augmented scheme. The update according to the scheme $\Delta \lambda_{T}^{L}=\Delta \lambda_{T}^{L-1}+\mathbf{B}\left(\boldsymbol{\xi}_{(I)}-\boldsymbol{\xi}_{(K-1)}\right)$ inside loop 2 allows to enforce sticking conditions similar to the normal penetration. The modification for large sliding problems is as follows: the sticking condition is satisfied in the penalty form, then the trial tangential traction $\mathbf{T}^{K, L, I}$ is computed as $\mathbf{T}^{K, L, I}=\mathbf{T}^{K-1, L, I}+\mathbf{B}\left(\boldsymbol{\xi}_{(I)}-\boldsymbol{\xi}_{(K-1)}\right)$. The Lagrange multiplier for the tangential traction vector $\Delta \lambda_{T}^{L-1}$ is not introduced and, therefore, an update loop 2 does not exist.

An important modification for symmetrization should be done for the returnmapping scheme, see Table 3, where the sliding force instead of eqn. (12) is computed via the augmented multiplier for the normal traction $\lambda_{N}^{L-1}$ as

$$
\mathbf{T}^{s l}=-\frac{\mathbf{B F T}^{t r}}{\sqrt{\text { BFT }^{t r} \cdot \mathbf{F B F T}}{ }^{t r}}\left|\lambda_{N}^{L-1}\right| .
$$

The yield function (6) is respectively modified as

$$
\Phi_{\lambda}=\sqrt{\mathbf{T}_{t r}^{K, L, I} \cdot \mathbf{F} \mathbf{T}_{t r}^{K, L, I}}-\left|\lambda_{N}^{L-1}\right| .
$$

This leads to a constant sliding force for the internal loop and, therefore, the 
1. Loop over load applied in incremental steps: $K=K+1$ initial condition for tangential traction $\mathbf{T}^{0,0,0}=0$ initialization of Lagrange multiplier $\Delta \lambda_{T}^{0}=0$

2. Loop over augmented multipliers: $L=L+1$
$\begin{array}{r}\text { 3. Iterative solution of } \\ \text { global equilibrium equations: } \\ \text { with tangential traction } \\ \mathbf{T}^{K, L, I}=\mathbf{T}^{K-1, L, I}+\Delta \lambda_{T}^{L-1}+\mathbf{B}\left(\boldsymbol{\xi}_{(I)}-\boldsymbol{\xi}_{(K-1)}\right) \\ \boldsymbol{\xi}_{(I)} \text { is a projection point in each iteration } I, \\ \boldsymbol{\xi}_{(K-1)} \text { is a projection point in the previous load } \\ \text { step. }\end{array}$

Update multipliers

$$
\Delta \lambda_{T}^{L}=\Delta \lambda_{T}^{L-1}+\mathbf{B}\left(\boldsymbol{\xi}_{(I)}-\boldsymbol{\xi}_{(K-1)}\right)
$$

Convergence is checked by proximity to the initial sticking point:

$$
\sqrt{a_{i j}\left(\xi_{(I)}^{i}-\xi_{(K-1)}^{i}\right)\left(\xi_{(I)}^{j}-\xi_{(K-1)}^{j}\right)} \leq \epsilon_{T}
$$

where $\epsilon_{T}$ - specified tolerance for tangential displacements

first main part of the sliding tangent matrix is zero $\mathbf{K}_{T, s l}^{m, 1}=0$. Then, the full tangent matrix for the sliding case becomes:

$$
\mathbf{K}_{s l}^{f u l l}=\mathbf{K}_{N}^{m}+\mathbf{K}_{N}^{r}+\mathbf{K}_{T, s l}^{m, 2}+\mathbf{K}_{T, s l}^{m, 3}+\mathbf{K}_{T, s l}^{r} .
$$

The part $\mathbf{K}_{T, s l}^{m, 3}$ is still nonsymmetric due to anisotropy. The matrix is fully symmetric only for isotropic friction. As it was found in numerical computations we can exclude this part with only a small loss of efficiency. This finally leads to the following matrix in the sliding case according to the inexact Uzawa algorithm:

$$
\mathbf{K}_{s l}^{f u l l}=\mathbf{K}_{N}^{m}+\mathbf{K}_{N}^{r}+\mathbf{K}_{T, s l}^{m, 2}+\mathbf{K}_{T, s l}^{r} .
$$

The tangent matrix for sticking remains symmetric:

$$
\mathbf{K}_{s t}^{f u l l}=\mathbf{K}_{N}^{m}+\mathbf{K}_{N}^{r}+\mathbf{K}_{T, s t}^{m}+\mathbf{K}_{T, s t}^{r} .
$$

\section{$6 \quad$ Numerical examples}

Two cases with different anisotropy have been selected to illustrate the convergence for the proposed approach: constant orthotropy and polar orthotropy on the plane. 
Table 3: Return-mapping scheme for the symmetric Augmented Lagrangian method

1. Loop over load applied in incremental steps: $K=K+1$

initial condition for tangential traction $\mathbf{T}^{0,0,0}=0$

initialization of Lagrange multiplier $\Delta \lambda_{T}^{0}=0$

initialization of Lagrange multiplier $\Delta \lambda_{N}^{0}=0$

2. Loop over load augmented multipliers: $L=L+1$

3. Iterative solution of

global equilibrium equations:

a) compute trial tangential traction $\mathbf{T}_{t r}^{K, L, I}$

(Table 2)

b) compute trial yield function $\Phi_{\lambda}$

$$
\Phi_{\lambda}=\sqrt{\mathbf{T}_{t r}^{K, L, I} \cdot \mathbf{F T}_{t r}^{K, L, I}}-\left|\lambda_{N}^{L-1}\right| .
$$

c) return-mapping: real tangential traction $\mathbf{T}^{K, L, I}$

$\mathbf{T}^{K, L, I}= \begin{cases}\mathbf{T}_{t r}^{K, L, I} & \text { if } \Phi_{\lambda}<0 \\ \mathbf{T}^{s l}=-\frac{\hat{\mathbf{T}}}{\sqrt{\hat{\mathbf{T}} \cdot \mathbf{F} \hat{\mathbf{T}}}}\left|\lambda_{N}^{L-1}\right| & \text { if } \Phi_{\lambda} \geq 0\end{cases}$

where $\hat{\mathbf{T}}=\mathbf{B F T}_{t r}^{K, L, I}$

\subsection{Small sliding problem. Constant orthotropy.}

We present here an example, which has been analyzed for the penalty based approach in [14]. The rectangular block, see Fig. 3, is considered on an orthotropic plane. The dimensions of the block are $10 \times 10 \times 4$ with linear elastic properties: Young's modulus $E=2.10 \cdot 10^{4}$ and Poisson ratio $\nu=0.3$, assumed within a consistent dimension system.

The case of constant orthotropy is defined by the spectral representation of both, the adhesion tensor $\mathbf{B}$, and the friction tensor $\mathbf{F}$ as follows:

$$
\begin{gathered}
\mathbf{B}=\left[b_{j}^{i}\right]=-\left[\begin{array}{ll}
\varepsilon_{1} \cos ^{2} \alpha+\varepsilon_{2} \sin ^{2} \alpha & \left(\varepsilon_{1}-\varepsilon_{2}\right) \sin \alpha \cos \alpha \\
\left(\varepsilon_{1}-\varepsilon_{2}\right) \sin \alpha \cos \alpha & \varepsilon_{1} \sin ^{2} \alpha+\varepsilon_{2} \cos ^{2} \alpha
\end{array}\right] . \\
\mathbf{F}=\left[f_{k}^{i}\right]=\left[\begin{array}{ll}
\frac{1}{\mu_{1}^{2}} \cos ^{2} \beta+\frac{1}{\mu_{2}^{2}} \sin ^{2} \beta & \left(\frac{1}{\mu_{1}^{2}}-\frac{1}{\mu_{2}^{2}}\right) \sin \beta \cos \beta \\
\left(\frac{1}{\mu_{1}^{2}}-\frac{1}{\mu_{2}^{2}}\right) \sin \beta \cos \beta & \frac{1}{\mu_{1}^{2}} \sin ^{2} \beta+\frac{1}{\mu_{2}^{2}} \cos ^{2} \beta
\end{array}\right] .
\end{gathered}
$$

The following parameters are taken for the computations: normal penalty 


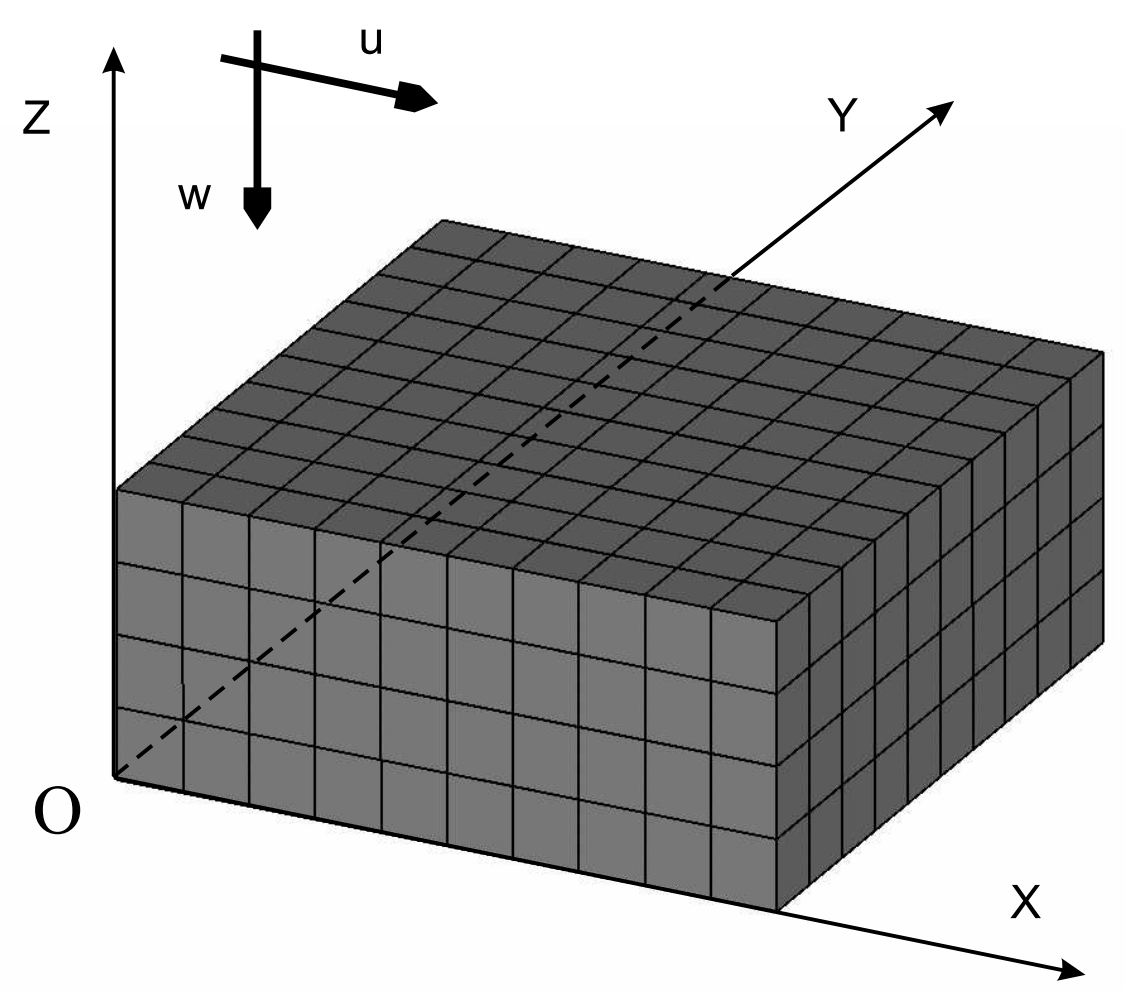

Figure 3: Geometry and loading of the rectangular in plane block.

parameter: $\varepsilon_{N}=2.1 \cdot 10^{4}$; adhesion parameters: $\varepsilon_{1}=3.0 \cdot 10^{4}, \varepsilon_{1}=2.0 \cdot 10^{4}$; friction coefficients: $\mu_{1}=3.0, \mu_{2}=2.0$; tolerance for penetration: $\epsilon_{N}=1.0$. $10^{-5}$; tolerance for tangential displacement: $\epsilon_{T}=1.0 \cdot 10^{-5}$; orthotropic angles: $\alpha=45^{\circ}, \beta=45^{\circ}$. This leads to a, so-called, geometrical isotropic case because of the ratio $\frac{\varepsilon_{1} \mu_{2}}{\varepsilon_{2} \mu_{1}}=1.0$.

The block (see Fig. 3) is located on the $X O Y$ plane and loaded by vertically prescribed displacements on the upper surface $w=1.0 \cdot 10^{-2}$. The penalty based approach gives convergence in 4 iterations, but both normal and tangential displacements inside the sticking region do not satisfy the prescribed tolerance. The results for the Augmented scheme are presented in Table 4. The method shows linear convergence as can be seen from the Tolerance column.

\subsection{Large sliding problem. Polar orthotropy.}

For the case with large sliding, we consider the block on a plane with polar orthotropy see [14]. An elastic block with dimensions $1 \times 1 \times 0.25$ and mesh $4 \times$ $4 \times 1$ is positioned on a rigid block, see Fig. 2. Linear elastic material is assumed within a consistent dimension system: Young's modulus $E=2.10 \cdot 10^{4}$; Poisson ratio $\nu=0.3$. The loading is applied sequentially by prescribing displacements on the upper surface in $(1+n)$ steps: 1$)$ vertical loading with $w=1.0 \cdot 10^{-2}$, 2) $n$ steps with horizontal displacement increments $\Delta u=1.0 \cdot 10^{-2}$ along the 
$X$ axis. The frictional tensor is isotropic with $\mu_{1}=\mu_{2}=0.2$, but the adhesion tensor has the following structure in the Cartesian coordinate system:

$$
\mathbf{B}=-\frac{1}{x^{2}+y^{2}}\left[\begin{array}{cc}
\varepsilon_{r} x^{2}+\varepsilon_{\varphi} y^{2} & \left(\varepsilon_{r}-\varepsilon_{\varphi}\right) x y \\
\left(\varepsilon_{r}-\varepsilon_{\varphi}\right) x y & \varepsilon_{r} y^{2}+\varepsilon_{\varphi} x^{2}
\end{array}\right] .
$$

Parameters for the adhesion tensor are chosen as $\varepsilon_{r}=1000, \varepsilon_{\varphi}=0.0$, in order to obtain the circular trajectory of the sliding block. Now only the normal tractions $N$ are updated and a tolerance for penetration $\epsilon_{N}=1.0 \cdot 10^{-5}$ is chosen. The gap for the penalty based approach is computed as $\xi^{3}=7.26 \cdot 10^{-3}$ if the normal penalty $\varepsilon_{N}=2.1 \cdot 10^{4}$ is chosen. The total number of iterations (4556) compared with the penalty approach (1834) is influenced only by the normal penetration. The final coordinates of the nodal point $X=0.292, Y=4.996$ are compared with the results focused with the penalty approach $X=0.306$, $Y=4.994$ in order to show that the main kinematical effect of the anisotropic surfaces is preserved for the Augmented Lagrangian method. A comparison of both trajectories leads to a slight difference comparable to the value of the initial penetration and the initial sticking displacements.

Table 4: Constant orthotropy on a plane. Convergence results for the symmetric Augmented Lagrangian method.

\begin{tabular}{|c|c|c|}
\hline Aug. No. & Number of eq. iter. & Tolerance \\
\hline 1 & 7 & $1.8742 \cdot 10^{-3}$ \\
\hline 2 & 14 & $6.0419 \cdot 10^{-4}$ \\
\hline 3 & 12 & $1.7536 \cdot 10^{-4}$ \\
\hline 4 & 12 & $4.0497 \cdot 10^{-5}$ \\
\hline 5 & 12 & $3.5972 \cdot 10^{-6}$ \\
\hline & $\sum 56$ & \\
\hline
\end{tabular}

\section{Conclusions}

In this contribution a symmetrization of the stiffness matrix has been developed within the Augmented Lagrangian method for anisotropic contact surfaces including both, anisotropy for adhesion and anisotropy for friction domains. It is shown that in general a fully coupled model necessarily leads to a fully nonsymmetric matrix in the case of sliding, but the covariant approach allows to estimate the structure of the tangent matrix part by part and, therefore, allows to construct a symmetric matrix used in accordance with the inexact Uzawa algorithm. However, as is shown, the Augmented Lagrangian method can not be directly applied to arbitrary anisotropic surfaces due to convergence problems. 
Thus, to create a robust algorithm, contact problems have to be subdivided into small sliding problems with low anisotropy where the distribution of the sticking-sliding zone is of interest, and into large sliding problems where the trajectory of the sliding body is of interest.

For the small sliding problems, both the normal, and the tangential contact tractions are augmented within the nested Uzawa algorithm. This makes it possible to enforce both normal and tangential sticking displacements to satisfy a prescribed tolerance. For the large sliding problems only the normal traction is augmented leading to the enforcement of only the normal displacements to satisfy a prescribe tolerance. In both cases the return-mapping scheme is exploited to obtain the real sliding tractions. Numerical examples including constant as well as nonlinear orthotropy e.g. a polar orthotropy showed the effectiveness of the proposed approach.

\section{References}

[1] Wriggers P (2002) Computational Contact Mechanics. John Wiley \& Sons.

[2] Laursen TA (2002) Computational Contact and Impact Mechanics. Fundamentals of Modeling Interfacial Phenomena in Nonlinear Finite Element Analysis Springer: New-York, Heidelberg, Paris.

[3] Harnau M, Konyukhov A, Schweizerhof K (2005) Algorithmic aspects in large deformation contact analysis using 'Solid-Shell' elements. Comput Struct 83:1804-1823.

[4] Puso MA, Laursen TA (2004) A mortar segment-to-segment contact method for large deformation solid mechanics, Comp Meth Appl Mech Engrg 193:601-629.

[5] Puso MA, Laursen TA (2004) A mortar segment-to-segment frictional contact method for large deformations Comp Meth Appl Mech Engrg 193:4891-4913.

[6] Fischer KA, Wriggers P (2005) Frictionless 2D Contact formulations for finite deformations based on the mortar method Comput Mech 36:226-244.

[7] Wohlmuth B (2000) A mortar finite element method using dual spaces for the Lagrange multiplier SIAM J Numer Anal 38:989-1012.

[8] Hüeber S, Stadler G, Wohlmuth B (2006) A primal-dual active set algorithm for three-dimensional contact problems with Coulomb friction. Preprint, Inst Angewandte Anal. und Num. Simulation, Stuttgart. 
[9] Bertsekas, D.P. (1982) Constrained optimization and Lagrange multiplier methods. New York: Acad. Pr.

[10] Fortin M., Glowinski, R. (1983) Augmented Lagrangian methods: applications to the numerical solutions of boundary-value problems. Amsterdam: North-Holland.

[11] Pietrzak G, Curnier A (1999) Large deformation frictional contact mechanics: continuum formulation and augmented Lagrangian treatment. Comp Meth Appl Mech Engrg 177:351-381.

[12] Laursen TA, Simo JC (1993) A continuum-based finite element formulation for the implicit solution of multibody, large deformation-frictional contact problems. Int J Numer Methods Engrg 36:3451-3485.

[13] Konyukhov A, Schweizerhof K (2006) Covariant description of contact interfaces considering anisotropy for adhesion and friction: Part 1. Formulation and analysis of the computational model. Comp Meth Appl Mech Engrg 196:103-117.

[14] Konyukhov A, Schweizerhof K (2006) Covariant description of contact interfaces considering anisotropy for adhesion and friction. Part 2. Linearization, finite element implementation and numerical analysis of the model. Comp Meth Appl Mech Engrg 196:289-303.

[15] Konyukhov A, Schweizerhof K (2004) Contact formulation via a velocity description allowing efficiency improvements in frictionless contact analysis. Comput Mech 33:165-173.

[16] Konyukhov A, Schweizerhof K (2005) Covariant description for frictional contact problems. Comput Mech 35:190-213.

[17] Konyukhov A, Schweizerhof K (2006) A special focus on 2D formulations for contact problems using a covariant description. Int $J$ Numer Methods Engrg 66:1432-1465.

[18] Stadler G (2004) Infinite-dimensional semi-smooth Newton and Augmented Lagrangian methods for friction and contact problems in elasticity. PhD Thesis, University of Graz, Graz. 